\title{
Serotonin in the pathophysiology and treatment of CNS disorders
}

\author{
Giuseppe Di Giovanni
}

Published online: 22 September 2013

(C) Springer-Verlag Berlin Heidelberg 2013

This special issue is in honour of Ennio Esposito, who devoted his unfortunately short but productive scientific career to serotonin research and made significant contributions to the field of neuropharmacology of monoaminergic systems in neuropsychiatric disorders.

After receiving his degree in Medicine at the University of Chieti, Ennio moved to Milan to the Laboratory of Neuropharmacology at the Institute of Pharmacological Research Mario Negri, under the supervision of Rosario Samanin. Successively, Ennio did his postdoc in the USA. at the Department of Psychiatry and Pharmacology at Yale University School of Medicine, New Haven, in the laboratory of Dr. Benjamin S. Bunney. Back in Italy, in 1989, he joined the Consorzio Mario Negri Sud, a Biomedical and Pharmacological Research Centre at Santa Maria Imbaro, Chieti. In this new institution, a few miles from his hometown, he created and directed the Laboratory of Neurophysiology for about 20 years.

The aim of this special issue is to present recent developments in research areas Ennio has been particularly involved in, that is, the serotonin receptors, especially the $5-\mathrm{HT}_{2 \mathrm{C}}$ subtype. It includes 18 papers written by several of his former students, co-workers and friends, as well as by distinguished international researchers with similar research interests.

G. Di Giovanni $(\square)$

Department of Physiology and Biochemistry,

Faculty of Medicine and Surgery, University of Malta,

Msida MSD 2080, Malta

e-mail: giuseppe.digiovanni@um.edu.mt;

digiovannig@cardfiff.ac.uk

G. Di Giovanni

Biomedical Building, Cardiff School of Biosciences,

Museum Avenue, Cardiff CF10 3US, UK

\section{Serotonin: still an enigma?}

Decades ago, in the 1950s, serotonin (5-hydroxytryptamine, 5-HT) was discovered in the mammalian central and peripheral nervous systems, and successively, its role as an essential neurotransmitter was revealed. Advances in understanding the effect of 5-HT have represented one of the success stories of neuropharmacology. Since its discovery, an enormous amount of experimental evidence has revealed the pivotal role of this biogenic amine in a bewildering diversity of behavioural and physiological processes. This is not surprising, considering the large distribution of 5-HT-containing axon terminals throughout the central nervous systems (CNS), although these monoamines are synthesized by a small group of neurons within the brain stem. The serotonergic system is one of the most diffusively organized projection systems of the mammalian brain. The majority of the neurons containing 5-HT generically called the raphe nuclei are located in the brainstem and in some regions of the reticular formation. Recent receptor discoveries have permitted the identification and classification of up to seven families of 5 -HT receptors $\left(5-\mathrm{HT}_{1}-5-\mathrm{HT}_{7}\right)$ to date. All 5-HT receptors belong to the seven transmembrane domain G-proteincoupled receptor (GPCR) superfamily, except for the 5- $\mathrm{HT}_{3}$ receptor which is a ligand-gated channel. The $5-\mathrm{HT}_{1}$ receptor class is comprised of five receptor subtypes $\left(5-\mathrm{HT}_{1 \mathrm{~A}}\right.$, $5-\mathrm{HT}_{1 \mathrm{~B}}, 5-\mathrm{HT}_{1 \mathrm{D}}, 5-\mathrm{HT}_{1 \mathrm{E}}$ and $5-\mathrm{HT}_{1 \mathrm{~F}}$ ), which, in humans, share $40-63 \%$ overall sequence identity and couple preferentially, although not exclusively, to Gi/o to inhibit cAMP formation.

Among the multiple classes of 5-HT receptors described in the CNS, much attention has been devoted to the 5- $\mathrm{HT}_{2}$ receptor family since it has been shown by experimental and clinical observation to represent a possible therapeutic 
target for the development of drugs for a range of CNS disorders such as schizophrenia, depression, drug abuse, eating disorders, $\mathrm{PD}$ and epilepsy. Indeed, 5- $\mathrm{HT}_{2}$ receptors are major targets for a wide array of psychoactive drugs, ranging from non-classical antipsychotic drugs, anxiolytics and anti-depressants, which have a $5-\mathrm{HT}_{2}$ antagonistic action, to hallucinogens, which are agonists of the $5-\mathrm{HT}_{2}$ receptors. Furthermore, recently, it has been shown that $5-\mathrm{HT}_{2}$ receptors have a potential significance in brain development, and in experience-dependent plasticity in the visual cortex. The $5-\mathrm{HT}_{2}$ receptor family forms a closely related subgroup of G-protein-coupled receptors and shows the typical heptahelical structure of an integral membrane protein monomer. They are currently classified as $5-\mathrm{HT}_{2 \mathrm{~A}}, 5-\mathrm{HT}_{2 \mathrm{~B}}$ and $5-\mathrm{HT}_{2 \mathrm{C}}$ subtypes, based on their close structural homology, pharmacology and signal transduction pathways, and they are differently localized in the brain. The amino acid sequence of the $5-\mathrm{HT}_{2}$ receptors shares a high degree $(>70 \%)$ of identity within the transmembrane segments; consequently, it is not surprising that many compounds bind with high affinity to all these three receptor subtypes. The 5- $\mathrm{HT}_{4}, 5-\mathrm{HT}_{6}$ and $5-\mathrm{HT}_{7}$ receptors have all been demonstrated to be preferentially coupled to Gs and promote cAMP formation. However, the sequence dissimilarity justifies the classification into different groups. Although the functional significance remains to be revealed, the interest in these receptor subtypes has increased after the demonstration that several antipsychotic drugs including clozapine, chlorpromazine, risperidone and olanzapine display a significant affinity for these sites. It appears likely, though, that $5-\mathrm{HT}_{6}$ receptors will have an important future role in the treatment for neuropsychiatric illnesses-especially in terms of enhancing cognition. Recent studies suggest that the $5-\mathrm{HT}_{7}$ receptor is involved in thermoregulation, circadian rhythm, learning and memory, hippocampal signalling, sleep and endocrine regulation. Thus, selective $5-\mathrm{HT}_{7}$ receptor ligands might prove therapeutically useful in the treatment of a number of neuropsychiatric disorders.

Two subtypes of the $5-\mathrm{HT}_{5}$ receptor $\left(5-\mathrm{HT}_{5 \mathrm{~A}}\right.$ and $5-\mathrm{HT}_{5 \mathrm{~B}}$ ), sharing $70 \%$ overall sequence identity, have been found in rodents, whereas only the $5-\mathrm{HT}_{5 \mathrm{~A}}$ subtype has been found in humans. Recent studies have shown that the human recombinant $5-\mathrm{HT}_{5 \mathrm{~A}}$ receptor activation produces an inhibition of cAMP production, indicating negative coupling to cAMP via Gi and Go; however, the receptor may also couple positively to cAMP. The human $5-\mathrm{HT}_{5 \mathrm{~B}}$ gene does not encode a functional protein because its coding sequence is interrupted by stop codons. The $5-\mathrm{HT}_{5 \mathrm{~B}}$ receptor is the first example of a brain-specific protein not found in human.

It is now well known that dysfunction of central serotonergic systems underlies the pathophysiology of some neuropsychiatric disorders, including depression, anxiety, schizophrenia, attention deficit hyperactivity disorders, drug abuse, Parkinson's disease (PD), Alzheimer's disease (AD) and Gilles de la Tourette's syndrome. Therefore, a better understanding of 5-HT systems, in normal and pathological conditions, will provide new pharmacological approaches for the treatment of these several neuropsychiatric disorders.

With this in mind, this special issue on "Serotonin in the pathophysiology and treatment of CNS disorders" offers as varied a picture as possible, by including articles across a wide range of disciplinary areas, together with different aspects of the 5-HT function in both physiological and pathological phenomena. This number of Experimental Brain Research seeks to provide an overview of the recent developments in our understanding of 5-HT pathophysiology, focusing on the role of 5- $\mathrm{HT}_{2}$ receptors in health and disease.

The selection begins with three contributions by HerrickDavis, Stam and Mengod, respectively, on new insights into serotonin receptor dimerization, genetic and biochemistry of $5-\mathrm{HT}_{2}$ receptors.

The next three articles focus on the role of serotonin in the hippocampus. Olvera-Cortés and colleagues review the serotonergic modulation of hippocampal theta activity in relation to hippocampal information processing. Bombardi and Di Giovanni focus instead their review on the role of $5-\mathrm{HT}_{2 \mathrm{~A}}$ receptors in the amygdala and hippocampus, and Di Giovanni and colleagues show that the activation of $5-\mathrm{HT}_{1 \mathrm{~A} / 7}$ receptors might be beneficial in the treatment for temporal lobe epilepsy and co-morbid cognitive impairments.

Studies in the third section further concentrate on different aspects of 5- $\mathrm{HT}_{2}$ receptor pathophysiology. Pessia and collaborators show that distinct actions of 5-HT, through $5-\mathrm{HT}_{2}$ receptors, are mediated by potassium $\mathrm{Kv}$ channels. Huot and Fox critically review studies assessing the SERT (5-HT transporter), as well as $5-\mathrm{HT}_{1 \mathrm{~A}}$ and $5-\mathrm{HT}_{2 \mathrm{~A}}$ receptors in idiopathic PD and animal models of PD, and discuss unmet challenges to effectively treat manifestations of PD using drugs specific for these targets. The following three contributions from Deurwaerdère and collaborators are further focused on serotonin modulation of basal ganglia activity. The first reviews the multiple controls exerted by $5-\mathrm{HT}_{2 \mathrm{C}}$ receptors upon basal ganglia function. In the second, they present the results of an electrophysiological study on the enhanced oral response to the $5-\mathrm{HT}_{2 \mathrm{C}}$ agonist Ro 60-0175 in Parkinsonian rats showing the involvement of the entopeduncular nucleus. In their final article, Deurwaerdère and colleagues investigate the role of $5-\mathrm{HT}_{2 \mathrm{C}}$ receptors in the enhancement of c-Fos expression induced by a $5-\mathrm{HT}_{2 \mathrm{~B} / 2 \mathrm{C}}$ inverse agonist and $5-\mathrm{HT}_{2}$ agonists in the rat basal ganglia. The contribution by Spampinato and colleagues provides an overview of the pivotal role of $5-\mathrm{HT}_{2 \mathrm{C}}$ 
in drug addiction, concentrating on cocaine effects. To investigate the effects of dorsomedial medullary 5-HT2 receptor antagonism on initial ventilatory airway responses to hypercapnic hypoxia, and to shed light on the mechanisms that mediate them, Kanamaru et al. performed a neurochemical study in mice. The experimental results showed that dorsomedial medullary $5-\mathrm{HT}_{2}$ activity elicits immediate onset of poikilocapnic hypoxic ventilatory airway responses, which are compensated for by hypercapnia.

The following section of the special issue comprises two studies relating to the most recently identified serotonin receptor, the 5- $\mathrm{HT}_{7}$. Clearly, the $5-\mathrm{HT}_{7}$ receptor may be of value as a novel therapeutic target, as highlighted by Van Craenenbroeck and colleagues. Nevertheless, the clinical utility of 5-HT receptor agents awaits the development of selective ligands. Some hope comes from Leopoldo and collaborators that synthesized compounds with improved affinity for $5-\mathrm{HT}_{7}$ receptor, and in parallel, they systematically evaluated the in vitro metabolic stability of the target compounds with the aim of gaining more insight into structural features that might improve pharmacokinetic properties.

The last section of the special issue deals with disorders such as depression, anxiety aggression and suicidality. Nakazato and colleagues by using wireless voltammetry show that dual modes of extracellular serotonin elevation in the ventral striatum may play a role in suppressing social stress. A slow phasic mode of serotonin increases caused adaptation to social stress; a drug-induced tonic mode of serotonin increases promoted adaptive changes in the phasic serotonin increases. These results imply a functional mechanism that may explain the efficacy of SSRI therapy for stress and anxiety disorders. Clinically, the authors hypothesized that the dual modes of serotonin elevation may play a role in inhibiting the vicious cycle in the neuronal circuit of patients with anxiety disorders. Using an in vivo combined temperature, blood flow, oximetric and electrochemical approach, Crespi tested the intriguing hypothesis that therapeutic manipulation of brain temperature may represent a key mechanism in the treatment for mood disorders. His results show that antidepressant bupropion has central thermogenic properties and confirm its positive influence on dopaminergic activity.

I have decided to conclude this special issue with the contribution of Boulougouris and colleagues, which is an example of integration of different research fields, psychology, behaviour and neuropharmacology dealing with suicide, the great man's retreat from life. Every year millions of people around the world try to kill themselves and nearly one million of them succeed. The authors review preclinical data with clinical practice and psychotherapy aiming to integrate some aspects of neurobiology of human suicidal behaviour into a model of suicide that can be used in a clinical encounter.

In conclusion, this special issue aims to cover the latest research, but for obvious reasons, it cannot be exhaustive given 5-HT complexity. Although 5-HT function in the brain has been extensively studied, much work remains to be done to clarify it role. The recent development of molecular and genetic approaches and the synthesis of more subtype-selective ligands for 5-HT receptors will not only allow a detailed understanding of 5-HT physiology but will also lead to the development of new treatment strategies, appropriate for those numerous neuropsychiatric disorders in which an alteration in 5-HT has been hypothesized.

I hope that the current issue has contributed towards answering some of the important questions in the serotonin field and will help bring about further dialogue between researchers from different fields, which is the only real way to finally reveal the serotonin enigma.

I would like to conclude my editorial remembering Ennio again, for his competent and enthusiastic approach to research, intellectual curiosity and his friendship that has had an impact upon my and many other researchers' lives. I am sure that Ennio would have enjoyed reading this fine special issue of Experimental Brain Research.

The following papers that have been written for this special issue have been or will be published elsewhere:

Quesseveur G, Repérant C, David DJ, Gardier AM, Sanchez C, Guiard BP. (2013) 5- $\mathrm{HT}_{2} \mathrm{~A}$ receptor inactivation potentiates the acute antidepressant-like activity of escitalopram: involvement of the noradrenergic system. Exp Brain Res. 226(2):285-295. doi:10.1007/s00221-0133434-3. (This paper was erroneously published in a regular volume).

The contributions from Ángel Pazos Carroand and Alvaro Diaz, György Bagdy, and Reza Rahimian will be published in regular issues of $E B R$ since at the time of the finalization of this special issue were still in production.

Acknowledgments My most hearty thanks go to Dr. Andrea Pillmann of Springer-Verlag, Heidelberg, and Bill Yate as assigned Managing Editor and Margrit Derrick, and the reviewers and all who contributed in one way or other in publishing this special issue. 